\title{
Expression and DNA methylation status of microRNA-375 in patients with type 2 diabetes mellitus
}

\author{
KAN SUN $^{1}$, XIANGYUN CHANG ${ }^{1}$, LIANG YIN $^{1}$, JUN LI $^{1}$, TING ZHOU ${ }^{2}$, CHEN ZHANG $^{1}$ and XUAN CHEN ${ }^{1}$ \\ ${ }^{1}$ Department of Endocrinology and Metabolism; ${ }^{2}$ The Central Laboratory, First Affiliated Hospital, \\ Shihezi University School of Medicine, Shihezi, Xinjiang 832002, P.R. China
}

Received July 2, 2013; Accepted December 6, 2013

DOI: $10.3892 / \mathrm{mmr} .2013 .1872$

\begin{abstract}
Recent studies have shown that DNA methylation in the promoter of microRNA-375 (miR-375) downregulates its expression during tumorigenesis. However, it is not known if $\mathrm{CpG}$ methylation of the $m i R-375$ promoter also has a role in the pathogenesis of type 2 diabetes mellitus (T2DM). In this study, the expression level and $\mathrm{CpG}$ methylation status of $m i R-375$ in patients with T2DM were analyzed. Plasma samples from 100 patients with T2DM and 100 healthy controls with normal glucose tolerance (NGT) were collected. The plasma levels of $m i R-375$ were examined using quantitative polymerase chain reaction (qPCR) and the methylation status of $17 \mathrm{CpG}$ sites in the promoter of the $m i R-375$ were determined using MassARRAY spectrometry. The plasma levels of $m i R-375$ were found to be upregulated in patients with T2DM compared with controls with NGT $(\mathrm{P}<0.05)$. Overall, the methylation levels of the $m i R-375$ promoter in patients with T2DM were not significantly different compared with controls with NGT; however, further studies revealed that four of the eight analyzed individual $\mathrm{CpG}$ units within the amplicon were significantly hypomethylated in T2DM samples compared with the NGT samples. This study demonstrated for the first time, to the best of our knowledge, that miR-375 is overexpressed in plasma in patients with T2DM, and this may be used as a novel biomarker to distinguish between patients with T2DM and healthy individuals. It was also demonstrated in this study that the miR-375 promoter is hypomethylated, in patients with T2DM, which may regulate the expression of $m i R-375$ and contribute to the pathogenesis of T2DM.
\end{abstract}

\section{Introduction}

Type 2 diabetes mellitus (T2DM) is a complex polygenic disease, commonly resulting from defects in insulin secretion

Correspondence to: Dr Kan Sun, Department of Endocrinology and Metabolism, First Affiliated Hospital, Shihezi University School of Medicine, Shihezi, Xinjiang 832002, P.R. China

E-mail: sunkan_shz86@126.com

Key words: miR-375, type 2 diabetes, methylation and diminished sensitivity of target tissues to insulin (1). $\beta$-cell dysfunction is a fundamental characteristic of T2DM development, and it leads to quantitative and qualitative abnormalities in insulin secretion, abnormalities in the kinetics of insulin secretion and progression of the defects with time (2).

MicroRNAs (miRNAs) are endogenous small non-coding RNAs ( 22 nucleotides), which are involved in post-transcriptional control of gene expression (3). They directly control the expression of a large portion of the human genome and are involved in the regulation of major cellular activities, including metabolism, differentiation, proliferation and apoptosis $(4,5)$. miRNAs have been suggested to have a role in multiple human diseases (6) and increasing evidence has shown that miRNAs are also involved in the pathogenesis of metabolic diseases, including diabetes mellitus. Previous studies have revealed that miRNAs, including $m i R-375, m i R-9$ and $m i R-124 a$, are important regulators of specialized $\beta$-cell functions (7-10). It has been shown that $m i R-375$, a pancreatic islet-specific miRNA, regulates insulin secretion through direct inhibition of insulin exocytosis (7). High levels of $m i R-375$ are found in the pancreatic islet of obese (ob/ob) mice and have been shown to regulate glucose homeostasis (8). One study demonstrated that upregulated pancreatic $m i R-375$ is a useful biomarker for pathways in the pathogenesis of T2DM associated with islet amyloid deposition and $\beta$-cell deficit (11). These findings suggest that $m i R-375$ regulates the function of $\beta$ cells in T2DM, but the exact role of miRNA-375 in T2DM has yet to be elucidated.

Previous studies have also investigated the role of epigenetics in the pathogenesis of T2DM $(12,13)$. Epigenetic modifications, particularly DNA hypermethylation, have been found to have a role in the regulation of genes important for protection against T2DM. miR-375 is encoded in the intergenic region and has an independent promoter containing $\mathrm{CpG}$ islands, which provides a basis for the regulation of its expression by methylation. Therefore, it was hypothesized that aberrant methylation of the human $m i R-375$ promoter may lead to abnormal expression, thereby causing $\beta$-cell dysfunction, and ultimately participate in the pathogenesis of the T2DM. This has already been previously demonstrated in certain types of cancer (14-16).

In China, the Kazak population has a significantly higher proportion of overweight individuals and a higher incidence of insulin resistance and hypertension compared with the 
Han population (17). Furthermore, the Kazak population are known to consume more meat and fewer vegetables than the Han population. However, the prevalence of T2DM in the Kazak population is only $3.65 \%$, which is lower compared with the Han population in the same region (17). This suggests that the Kazak population may have a unique genetic background, and was the basis for the investigations in this study. It was hypothesized that the expression level and $\mathrm{CpG}$ methylation status of miR-375 may be important in the incidence and severity of T2DM. The changes in $m i R-375$ expression level and the quantitative methylation status of $\mathrm{CpG}$ within the $m i R-375$ promoter were assessed to determine whether aberrant promoter methylation of $m i R-375$ was present in patients with T2DM. The findings may lead to the identification of a novel biomarker for the diagnosis of T2DM.

\section{Materials and methods}

Subjects. This study was prospectively performed and approved by the Institutional Ethics Committees of the First Affiliated Hospital of Shihezi University School of Medicine (Shihezi, Xinjiang, China) and conducted in accordance with the ethical guidelines of the Declaration of Helsinki. Written informed consent was obtained from all individuals prior to the start of the study. Individuals of Chinese Kazak origin with T2DM $(n=100)$ and healthy controls with normal glucose tolerance (NGT; $n=100$ ) were recruited from the Department of Endocrinology and Metabolism at the First Affiliated Hospital of Shihezi University School of Medicine between 2010 and 2011. Diagnosis of T2DM was based on the World Health Organization criteria as fasting glucose levels $\geq 7 \mathrm{mmol} / \mathrm{l}$ (126 mg/dl), $2 \mathrm{~h}$ oral glucose tolerance test glucose levels $\geq 11.1 \mathrm{mmol} / 1$ (200 mg/dl) or clinical diagnosis of the disease. Patients with T2DM (54 male and 46 female; mean age, $51.33 \pm 11.75$ years) were recruited when they were hospitalized for treatment for poor glycemic control. Individuals who came for a health checkup in the hospital were recruited as healthy controls (44 male and 56 female; mean age, $48.55 \pm 12.41$ years). Any individuals who may have had an infectious disease prior to or during the recruitment were excluded from this study, as well as patients with autoimmune diseases.

Nucleic acid isolation. RNAs were isolated from peripheral plasma samples from patients with T2DM and controls with NGT using the miRNeasy Mini kit 50 (Qiagen, Hilden, Germany) and genomic DNA was isolated from peripheral plasma using the DNeasy Blood and Tissue kit (Qiagen) in accordance with the manufacturer's instructions. The RNA and DNA were quantified by measuring their absorption at 260 nm (Toption Instrument Co., Ltd., Xi'an, China).

Reverse transcription and quantitative polymerase chain reaction ( $q P C R$ ). The TaqMan ${ }^{\circledR}$ microRNA Reverse Transcription kit (Applied Biosystems ${ }^{\circledR}$, Foster City, CA, USA) was used for complementary DNA synthesis. For reverse transcription, $10 \mu 1$ TaqMan Universal PCR Master Mix (Applied Biosystems) and $5 \mu 1$ total RNA were reverse transcribed at $16^{\circ} \mathrm{C}$ for $30 \mathrm{~min}, 42^{\circ} \mathrm{C}$ for $30 \mathrm{~min}$, and $85^{\circ} \mathrm{C}$ for $5 \mathrm{~min}$. $\mathrm{miR}-375$ expression was quantified in triplicate using qPCR using the ABI Prism 7300 Sequence Detection
System (Applied Biosystems). Taqman primers and probes for the target gene miR-375 and the internal control miRNA-16, which is often used as a normalization control for microRNAs, were obtained from Applied Biosystems. PCR was performed under the following conditions: $50^{\circ} \mathrm{C}$ for 2 min then $95^{\circ} \mathrm{C}$ for $10 \mathrm{~min}$, followed by 40 cycles at $95^{\circ} \mathrm{C}$ for $15 \mathrm{sec}$ and $60^{\circ} \mathrm{C}$ for 1 min. miRNA-16 was used as an internal control to normalize the expression level of each sample. The comparative threshold cycle $(\mathrm{Ct})$ method was used to evaluate the relative abundance of miR-16 compared with U6 expression (fold changes relative to miRNA-16).

Analysis of methylation. To quantify the levels of methylation of the $\mathrm{CpG}$ islands in the miR-375 promoter, the high-throughput MassARRAY platform (Sequenom Inc., San Diego, CA, USA) was used as previously described (18). Briefly, bisulfite-treated DNA was amplified using primers for the $m i R-375 \mathrm{CpG}$ island. The primers were designed using EpiDesigner (Sequenom Inc.) and were as follows: forward 5'-aggaagagagGGGTGGAGTATTTTTGTTTGTTG-3' and reverse 5'-cagtaatacgactcactatagggagaaggct AAAAACATAATCCAAAACATCCT AAT-3'. The PCR products were spotted on a 384-pad SpectroCHIP (Sequenom Inc.), followed by spectral acquisition on a MassARRAY Analyzer. Methylation data of the individual units (1-3 CpG sites/unit) were generated using the EpiTyper version 1.0 .5 software (Sequenom Inc.).

Statistical analysis. The differences in miR-375 CpG methylation between patients with T2DM and controls with NGT were assessed using the Mann-Whitney and Kruskal-Wallis tests. The distances between $\mathrm{CpG}$ methylation sites to transcription start sites were calculated using the RMySQL package and the SQL database version of the University of California Santa Cruz (UCSC) genome browser (http://genome.ucsc. edu/cgi-bin/hgGateway). Two-dimensional clustering was performed with the heatmap. 2 function in the gregmisc package using the $\mathrm{R}$ statistical environment. Classical multidimensional scaling was performed using the cmdscale function and visualization was conducted through the scatter plot3d function in the gregmisc package. A Student's t-test, a Wilcox test or a Fisher's exact test was utilized to compare the qPCR results in different groups with standard function in $\mathrm{R}$ statistical environment. $\mathrm{P}<0.05$ was considered to indicate a statistically significant difference.

\section{Results}

Characteristics of the subjects. A total of 100 patients with T2DM and 100 age-matched healthy controls with NGT participated in this study. The clinical characteristics of the subjects are shown in Table I. The average age was $51.33 \pm 11.75$ years for patients with T2DM and $48.55 \pm 12.41$ years for controls with NGT. As expected, the patients with T2DM had a higher body mass index (BMI), waist-to-hip ratio (WHR), systolic blood pressure (SBP), fasting plasma glucose (FPG), low-density lipoprotein cholesterol (LDL-C), total cholesterol (TC) and triglyceride (TG) and lower highdensity lipoprotein cholesterol (HDL-cholesterol) compared with controls with NGT. 
Table I. Characteristics of the study subjects.

\begin{tabular}{lccr}
\hline & NGT & T2DM & P-value \\
\hline Gender (male/female) & $44 / 56$ & $54 / 46$ & 0.159 \\
Age (years) & $48.55 \pm 12.41$ & $51.33 \pm 11.75$ & 0.105 \\
WHR & $0.88 \pm 0.04$ & $0.92 \pm 0.05$ & 0.017 \\
BMI $\left(\mathrm{kg} / \mathrm{m}^{2}\right)$ & $24.44 \pm 4.63$ & $26.30 \pm 4.08$ & 0.005 \\
SBP $(\mathrm{mmHg})$ & $129.90 \pm 23.72$ & $141.46 \pm 17.89$ & $<0.001$ \\
DBP $(\mathrm{mmHg})$ & $83.81 \pm 13.33$ & $86.18 \pm 10.88$ & 0.196 \\
FPG $(\mathrm{mmol} / \mathrm{l})$ & $4.10 \pm 0.64$ & $10.60 \pm 4.27$ & $<0.001$ \\
TC $(\mathrm{mmol} / \mathrm{l})$ & $3.67 \pm 0.92$ & $5.10 \pm 1.39$ & $<0.001$ \\
TG $(\mathrm{mmol} / \mathrm{l})$ & $1.02 \pm 0.69$ & $1.90 \pm 1.42$ & $<0.001$ \\
LDL-C (mmol/l) & $1.73 \pm 0.58$ & $2.86 \pm 1.03$ & $<0.001$ \\
HDL-C $(\mathrm{mmol} / \mathrm{l})$ & $1.33 \pm 0.45$ & $1.10 \pm 0.24$ & $<0.001$ \\
\hline
\end{tabular}

NGT, normal glucose tolerance; T2DM, type 2 diabetes mellitus; WHR, waist-to-hip ratio; BMI, body mass index; SBP, systolic blood pressure; DBP, diastolic blood pressure; FPG, fasting plasma glucose; TC, total cholesterol; TG, triglyceride; LDL-C, low-density lipoprotein cholesterol; HDL-cholesterol, high-density lipoprotein cholesterol.

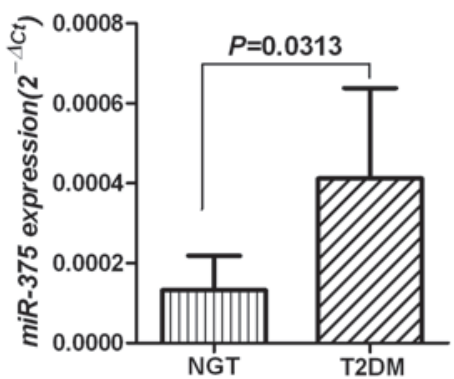

Figure 1. Levels of $m i R-375$ expression in NGT and T2DM samples. Plasma mRNA levels of $m i R-375$ in 100 patients with T2DM and 100 controls with NGT were determined using quantitative polymerase chain reaction (qPCR) as described in Materials and methods. The relative expression values were defined as the expression ratio of $m i R-375$ to $m i R-16$. The values are presented as the mean \pm standard deviation (SD) from three independent experiments. The significance level was determined using a Student's t-test between the the two groups and $\mathrm{P}<0.05$ was considered to indicate a statistically significant difference. miR-375, microRNA-375; NGT, normal glucose tolerance; T2DM, type 2 diabetes mellitus.

Upregulation of miR-375 expression in patients with T2DM compared with controls with NGT. It has previously been demonstrated that $m i R-375$ expression is lost in different types of cancer $(15,16)$; therefore it was investigated in this study whether aberrant expression of $m i R-375$ was present in the patients with T2DM. qPCR was performed to determine the expression levels of $m i R-375$ mRNA in T2DM plasma samples and corresponding NGT samples. As shown in Fig. 1, the level of miR-375 mRNA was significantly upregulated in T2DM samples compared with the NGT samples $(\mathrm{P}=0.0313)$.

Specific CpG hypomethylation of the miR-375 promoter may contribute to its overexpression in patients with T2DM. In order to understand the mechanism of $m i R-375$ upregulation, the methylation status of the promoter region of $m i R-375$ was investigated. To determine whether the methylation status was correlated with the upregulation of $m i R-375$, T2DM and corresponding NGT samples were analyzed using MassARRAY
EpiTyper. Hierarchical clustering identified substantial differences in the quantitative methylation profiling of T2DM samples compared with controls (Fig. 2).

A schematic representation of the promoter region of miR-375 according to UCSC Genome Browser's CpG island annotations and miRbase release 13.0 is shown in Fig. 3. Methylation was present from -990 to $-1,258$ bp relative to the transcription start site of $m i R-375$ in T2DM samples. Eight $\mathrm{CpG}$ units (comprising a total of $17 \mathrm{CpG}$ sites) spanning $267 \mathrm{bp}$ in the specified promoter region of $m i R-375$ were analyzed in 100 T2DM samples and 100 controls (a total of 31,248 sites in all analyzed samples). The overall methylation of the $m i R-375$ promoter was found to be $<10 \%$ for the T2DM and NGT samples; the mean levels of miR-375 methylation, calculated from the methylation levels of the $17 \mathrm{CpG}$ residues, were $8.59 \%$ in the patients with T2DM and $8.97 \%$ in the control group with NGT ( $\mathrm{P}=0.9582$; Fig. 4). The levels of miR-375 promoter methylation varied between 4.6 and $15.3 \%$ in T2DM samples and between 3.0 and $17.9 \%$ in NGT samples.

Although no differences in overall methylation levels between control and T2DM samples were observed, hypomethylation of specific critical $\mathrm{CpG}$ units was investigated to determine whether they accounted for the increased miR-375 expression observed in T2DM samples. Analysis of the individual $\mathrm{CpG}$ units in T2DM samples and controls using MassARRAY EpiTyper revealed that six CpG units in this amplicon were hypomethylated, with the exception of $\mathrm{CpG}_{-} 7$ and $\mathrm{CpG}$ _20. In addition, significant differences were observed in four specific $\mathrm{CpG}$ units, $\mathrm{CpG}_{-} 1.2, \mathrm{CpG}_{\mathbf{1}} 3.4$, CpG_5.6 and CpG_21.22.23.34, which were hypomethylated in T2DM samples but not in NGT samples (Fig. 5).

\section{Discussion}

Previous studies have shown that expression levels of $m i R-375$ have different effects in different types of cancer. For example, the upregulation of $m i R-375$ stimulates cell proliferation in breast and prostate cancer $(14,19)$; however, downregulation 


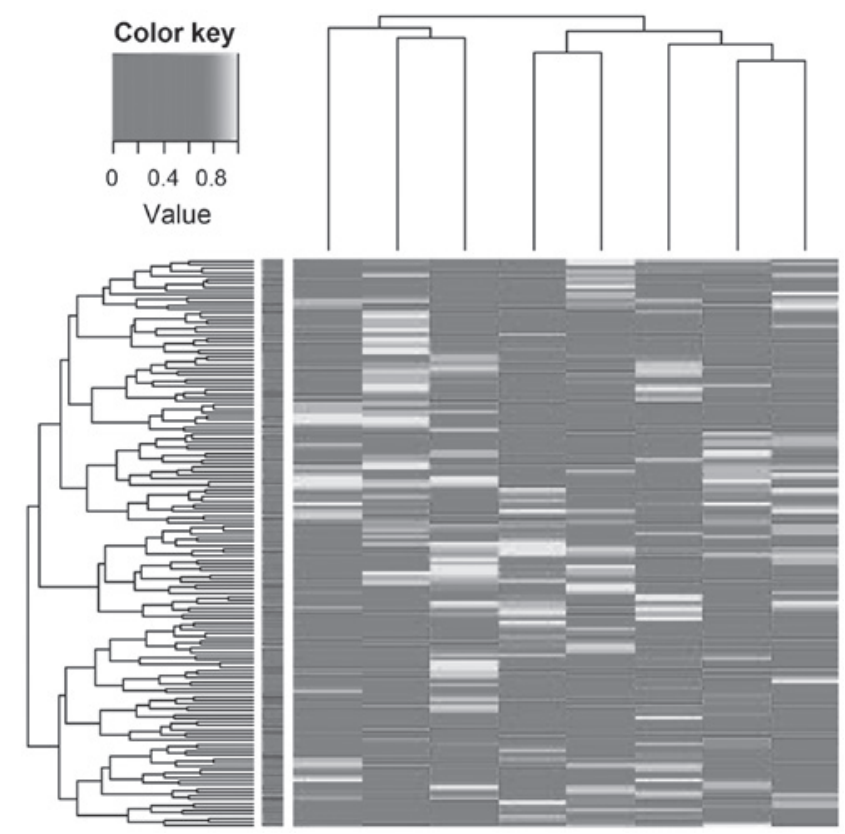

Figure 2. Hierarchical clustering of miR-375 methylation profiles in T2DM samples and NGT controls. MassARRAY (Sequenom Inc., San Diego, CA, USA) analysis was used to quantify DNA methylation of miR-375 in T2DM samples and NGT controls. Each row represents a sample and each column represents a CpG unit, which consists of a single CpG site or a combination of CpG sites. miR-375, microRNA-375; T2DM, type 2 diabetes mellitus; NGT, normal glucose tolerance.

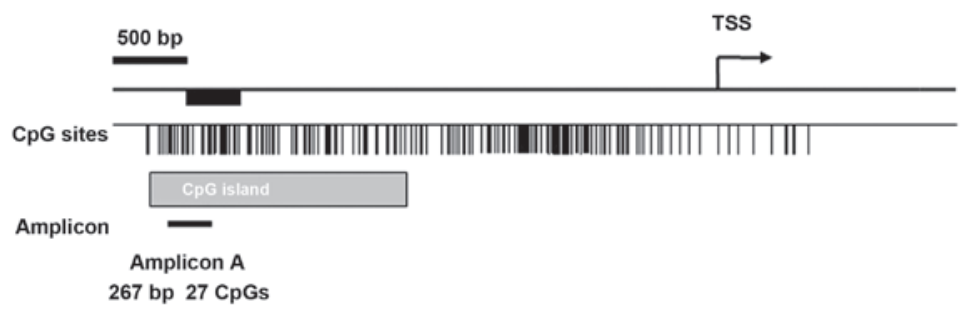

Figure 3. Schematic representation of the miR-375 promoter region. Vertical lines represent the CpG dinucleotides. The arrow indicates the transcriptional start site. The $\mathrm{CpG}$ island is represented by the gray box and the black-filled bars represents the MassARRAY-studied amplicon regions. Amplicon characteristics are shown beneath the black bars. bp, base pair; TSS, transcriptional start site; miR-375, microRNA-375.

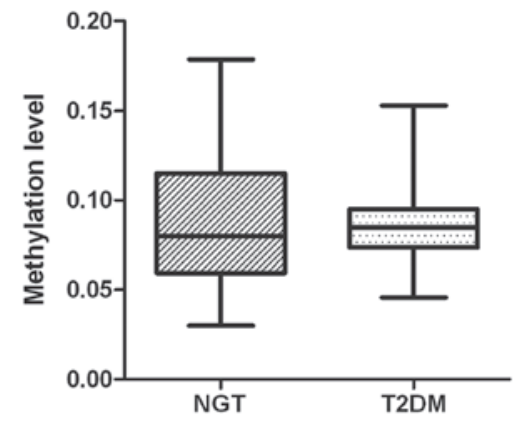

Figure 4. DNA methylation levels of $m i R-375$ promoters in the samples from patients with T2DM and NGT controls. MassARRAY (Sequenom Inc., San Diego, CA, USA) was used to determine the overall methylation levels. The mean methylation level of the $17 \mathrm{CpG}$ residues in the 100 samples was used to represent the promoter methylation levels. Data are presented as the mean \pm standard deviation $(\mathrm{SD})$. miR-375, microRNA-375; T2DM, type 2 diabetes mellitus; NGT, normal glucose tolerance.

of $m i R-375$ in gastric cancer leads to the activation of the phosphoinositide-dependent kinase 1-Akt (PDK1-Akt) and Janus kinase (JAK) oncogenic pathways $(15,20)$. In this study,

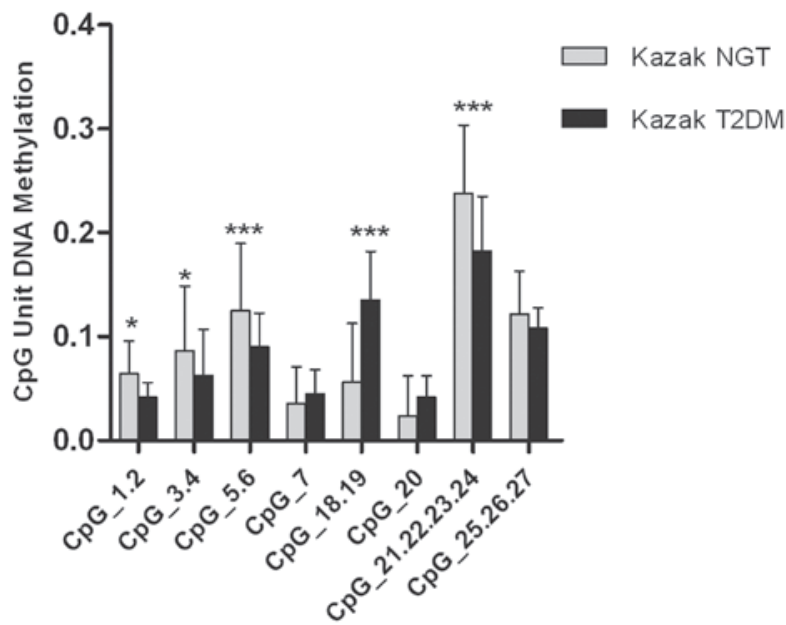

Figure 5. Specific miR-375 CpG methylation in T2DM samples compared with NGT samples. The average methylation of specific $\mathrm{CpG}$ units of amplicon in 100 patients with T2DM and 100 controls with NGT was determined using MassARRAY EpiTyper (Sequenom Inc., San Diego, CA, USA). The values represent the mean \pm standard error. Mann-Whitney and Kruskal-Wallis tests were used for the comparison between the two groups. ${ }^{* * * *} \mathrm{P}<0.001$; ${ }^{*} \mathrm{P}<0.05$, comparison between T2MD and NGT smaples. miR-375, microRNA-375; T2DM, type 2 diabetes mellitus; NGT, normal glucose tolerance. 
the expression pattern of $m i R-375$ was investigated in patients with T2DM. It was found that the plasma level of $m i R-375$ was significantly upregulated in T2DM samples compared with NGT samples (Fig. 1). It has previously been shown that overexpression of $m i R-375$ downregulates the expression of myotrophin, a pivotal factor implicated in the regulation of a variety of genes responsible for cellular apoptosis and proliferation (21). Together, these observations suggest that $m i R-375$ is involved in the pathogenesis of T2DM. Since plasma/serum miRNA levels are particularly stable, and the expression level of miRNAs in the serum is reproducible and consistent among individuals, a previous study investigated the presence of circulating miRNAs and their potential use as disease biomarkers (22). The results of the present study suggest that the plasma level of $m i R-375$ may allow patients with T2DM to be distinguished from healthy controls, and, therefore, $m i R-375$ may be used as a novel biomarker for T2DM.

Epigenetic modifications of DNA, such as methylation, have been suggested to have a key role in T2DM disease progression $(12,13)$, and miRNA are epigenetically regulated by DNA methylation (23). Several studies had revealed that the expression of $m i R-375$ is epigenetically regulated in oesophageal squamous cell carcinoma, as well as gastric and breast cancer (14-16); however, $m i R-375$ promoter methylation has not been directly linked to T2DM. In the present study, it was investigated whether the upregulation of $m i R-375$ was mediated by epigenetic mechanisms in patients with T2DM. Using MALDI-TOF MS, methylation patterns were evaluated at multiple $\mathrm{CpG}$ sites within the promoter regions of $m i R-375$, and it was found that overall there was less methylation in T2DM samples compared with NGT samples, although this difference was not statistically significant ( $\mathrm{P}>0.05)$ (Fig. 4). Since there is considerable variation between methylation levels of different $\mathrm{CpG}$ units, the methylation status of specific $\mathrm{CpG}$ units was further evaluated. The results revealed that there was a significant decrease in methylation in four specific CpG units in T2DM samples compared with NGT samples. These findings suggest that $m i R-375 \mathrm{CpG}$ island hypomethylation may be negatively correlated with $m i R-375$ expression. In addition, hypomethylation of $m i R-375$ may be used to predict the progression of T2DM. $m i R-375$ inhibits insulin secretion and decreases the number of $\beta$ cells. $m i R-375$ may also affect cell proliferation, differentiation and the expression of apoptosis-associated genes so as to promote the occurrence of apoptosis $(15,16)$. Therefore, it was hypothesized that hypomethylation of the miR-375 gene promoter may be involved in the development of diabetes.

In this study, aberrant $m i R-375$ expression and methylation were detected in the plasma of patients with T2DM. This has a potential clinical application for the identification of pateints with T2DM from individuals with NGT. Despite the small sample size, this is the first study, to the best of our knowledge, to report an alteration in the level of miR-375 in plasma of patients with T2DM. Clinical detection of upregulated miR-375 levels may not be limited to using plasma for T2DM diagnosis, and it may be interesting to investigate whether miR-375 is also present in other body fluids, for example saliva or urine, in order to establish a general diagnostic method.

In conclusion, it was demonstrated in this study that $m i R-375$ expression was significantly upregulated and hypomethylation was found in four specific $\mathrm{CpG}$ units of the miR-375 promoter in patients with T2DM compared with controls with NGT. These results suggest that DNA methylation may have an important role in the control of T2DM. These results may also lead to the development of miRNA-based targeted approaches for the treatment of T2DM.

\section{Acknowledgements}

This study was supported by the National Natural Science Foundation of China (81060065/H0711).

\section{References}

1. Butler AE, Janson J, Bonner-Weir S, Ritzel R, Rizza RA and Butler PC: Beta-cell deficit and increased beta-cell apoptosis in humans with type 2 diabetes. Diabetes 52: 102-110, 2003.

2. Guillausseau PJ, Meas T, Virally M, Laloi-Michelin M, Médeau V and Kevorkian JP: Abnormalities in insulin secretion in type 2 diabetes mellitus. Diabetes Metab 34 (Suppl 2): S43-S48, 2008.

3. Ambros V: The functions of animal microRNAs. Nature 431: 350-355, 2004.

4. Bartel DP: MicroRNAs: target recognition and regulatory functions. Cell 136: 215-233, 2009.

5. Uhlmann S, Zhang JD, Schwäger A, Mannsperger H, Riazalhosseini Y, Burmester S, et al: miR-200bc/429 cluster targets PLCgammal and differentially regulates proliferation and EGF-driven invasion than miR-200a/141 in breast cancer. Oncogene 29: 4297-4306, 2010.

6. Chang TC and Mendell JT: microRNAs in vertebrate physiology and human disease. Annu Rev Genomics Hum Genet 8: 215-239, 2007.

7. Poy MN, Eliasson L, Krutzfeldt J, Kuwajima S, Ma X, Macdonald PE, et al: A pancreatic islet-specific microRNA regulates insulin secretion. Nature 432: 226-230, 2004.

8. Poy MN, Hausser J, Trajkovski M, Braun M, Collins S, Rorsman P, et al: miR-375 maintains normal pancreatic alphaand beta-cell mass. Proc Natl Acad Sci USA 106: 5813-5818, 2009.

9. Baroukh N, Ravier MA, Loder MK, Hill EV, Bounacer A, Scharfmann R, et al: MicroRNA-124a regulates Foxa2 expression and intracellular signaling in pancreatic beta-cell lines. J Biol Chem 282: 19575-19588, 2007.

10. Plaisance V, Abderrahmani A, Perret-Menoud V, Jacquemin P, Lemaigre $F$ and Regazzi R: MicroRNA-9 controls the expression of Granuphilin/Slp4 and the secretory response of insulin-producing cells. J Biol Chem 281: 26932-26942, 2006.

11. Zhao H, Guan J, Lee HM, Sui Y, He L, Siu JJ, et al: Up-regulated pancreatic tissue microRNA-375 associates with human type 2 diabetes through beta-cell deficit and islet amyloid deposition. Pancreas 39: 843-846, 2010.

12. Gardner RJ, Mackay DJ, Mungall AJ, Polychronakos C, Siebert R, Shield JP, et al: An imprinted locus associated with transient neonatal diabetes mellitus. Hum Mol Genet 9: 589-596, 2000.

13. Ling C, Del Guerra S, Lupi R, Rönn T, Granhall C, Luthman H, et al: Epigenetic regulation of PPARGC1A in human type 2 diabetic islets and effect on insulin secretion. Diabetologia 51: 615-622, 2008.

14. de Souza Rocha Simonini P, Breiling A, Gupta N, Malekpour M, Youns M, Omranipour R, et al: Epigenetically deregulated microRNA-375 is involved in a positive feedback loop with estrogen receptor alpha in breast cancer cells. Cancer Res 70: 9175-9184, 2010

15. Tsukamoto Y, Nakada C, Noguchi T, Tanigawa M, Nguyen LT, Uchida T, et al: MicroRNA-375 is downregulated in gastric carcinomas and regulates cell survival by targeting PDK 1 and 14-3-3zeta. Cancer Res 70: 2339-2349, 2010.

16. Kong KL, Kwong DL, Chan TH, Law SY, Chen L, Li Y, et al: MicroRNA-375 inhibits tumour growth and metastasis in oesophageal squamous cell carcinoma through repressing insulin-like growth factor 1 receptor. Gut 61: 33-42, 2012.

17. Yang YN, Xie X, Ma YT, Li XM, Fu ZY, Ma X, et al: Type 2 diabetes in Xinjiang Uygur Autonomous Region, China. PLoS One 7: e35270, 2012. 
18. Lin HJ, Zuo T, Lin CH, Kuo CT, Liyanarachchi S, Sun S, et al: Breast cancer-associated fibroblasts confer AKT1-mediated epigenetic silencing of cystatin $\mathrm{M}$ in epithelial cells. Cancer Res 68: 10257-10266, 2008.

19. Szczyrba J, Nolte E, Wach S, Kremmer E, Stöhr R, Hartmann A, et al: Downregulation of Sec23A protein by miRNA-375 in prostate carcinoma. Mol Cancer Res 9: 791-800, 2011.

20. Ding L, Xu Y, Zhang W, Deng Y, Si M, Du Y, Yao H, et al: MiR-375 frequently downregulated in gastric cancer inhibits cel proliferation by targeting JAK2. Cell Res 20: 784-793, 2010.

21. Li Y, Xu X, Liang Y, Liu S, Xiao H, Li F, et al: miR-375 enhances palmitate-induced lipoapoptosis in insulin-secreting NIT-1 cells by repressing myotrophin (V1) protein expression. Int J Clin Exp Pathol 3: 254-264, 2010.
22. Mitchell PS, Parkin RK, Kroh EM, Fritz BR, Wyman SK, Pogosova-Agadjanyan EL, et al: Circulating microRNAs as stable blood-based markers for cancer detection. Proc Natl Acad Sci USA 105: 10513-10518, 2008.

23. Weber B, Stresemann C, Brueckner B and Lyko F: Methylation of human microRNA genes in normal and neoplastic cells. Cell Cycle 6: 1001-1005, 2007. 